\title{
PERFIL EPIDEMIOLÓGICO DA LEISHMANOSE VISCERAL HUMANA NAS REGIÕES DE SAÚDE DO ESTADO DE PERNAMBUCO NO PERÍODO DE 2004 A 2013.
}

\author{
Efraim Naftali Lopes SOARES ${ }^{1}$ \\ Filipe Antônio Lemos de LIMA $^{2}$ \\ Antonio Roberval Maciel da SILVA ${ }^{3}$ \\ Joice Camila Rodrigues e SILVA ${ }^{4}$ \\ André Carneiro COIMBRA ${ }^{5}$ \\ Laryssa Paes ALMEIDA ${ }^{6}$
}

\author{
${ }^{1}$ Biomédico. Especialista em Gestão de Sistemas e Serviços de Saúde (CpqAM), Gerente de Vigilância em Saúde da IV \\ Gerência Regional de Saúde (GERES). \\ ${ }^{2}$ Fisioterapeuta. Especialista em Cardiorrespiratória pelo Instituto Dante Pazzanese de Cardiologia- SP. \\ ${ }^{3}$ Sanitarista. Secretário de Saúde de Agrestina-PE \\ ${ }^{4}$ Biomédica. Especialista em Gestão das Clínicas pelo Hospital Sírio Libanez -SP. \\ ${ }^{5}$ Biomédico. Especialista em Microbiologia pela Faculdade ASCES. \\ ${ }^{6}$ Biomédica. Apoiadora do Programa de Enfrentamento as Doenças Negligenciadas (SANAR) IV GERES-PE.
}

Endereço para correspondência do autor: Rua: Bahia no 737, Bairro: Divinópolis, Caruaru-PE. CEP: 55010350. Email: efraimnaftali@gmail.com

Recebido em: 29/08/2015 - Aprovado em: 21/05/2016 - Disponibilizado em: 30/07/2016

\begin{abstract}
RESUMO:
Esse estudo buscou realizar um levantamento epidemiológico da Leishmaniose Visceral Humana (LVH) em Pernambuco, através do número de casos humanos no período de 2004 a 2013, distribuídos nas 12 Regiões de Saúde do Estado. Os dados coletados foram obtidos no Sistema de Informação de Agravos de Notificação Compulsória (SINANNET) da Secretaria Estadual de Saúde (SES/PE), através do levantamento epidemiológico dos casos notificados e confirmados da doença e o seu Coeficiente de Incidência (CI). Os resultados mostraram que, 2.255 casos foram notificados e confirmados registraram-se 863 de LVH, no período em estudo. No início da série histórica o CI foi de 2,23 casos/100.000 habitantes. No final, esse percentual declinou para 1,49. A partir do estudo, foi constatado que a Leishmaniose está presente em todo território com os maiores índices no Agreste e no Sertão pernambucano, sendo necessários investimentos nesta endemia para um melhor controle da doença.
\end{abstract}

Palavras-chave: Leishmaniose, Incidência, Epidemiologia.

\begin{abstract}
:
This study sought to conduct an epidemiological survey of Human Visceral Leishmaniasis (HVL) in the state of Pernambuco in Brazil, through the number of human cases from 2004 to 2013, distributed in 12 state regions of Health. The collected data were obtained from the Information System Diseases of Compulsory Notification (SINAN-NET) of the Health Department (SES / PE), through epidemiological survey from reported cases and confirmed cases of the disease, and its incidence ratio (CI). The results show that 2,255 cases of HVL were reported but just 863 were confirmed in the study period. At the beginning of the series time, the CI was 2.23 cases / 100,000 inhabitants. At the end, this percentage dropped to 1.49. From the study, it conclude that Leishmaniasis is present throughout the territory with the highest rates in the Agreste of Pernambuco, requiring investments in this endemic area for better control of the disease.
\end{abstract}

Keywords: Incidence, Leishmaniasis, Epidemiology. 


\section{INTRODUÇÃO}

As leishmanioses são doenças infecciosas que acometem o homem e outros animais vertebrados como: o cão (canis familiaris) no ambiente doméstico, bem como, no silvestre através das raposas (Dusicyon vetulus e Cerdocyon thous) causadas por várias espécies de protozoários do gênero Leishmania (ordem Kinetoplastidae, família Trypanosomatidae) e transmitidas por mosquitos da subfamília Phlebotominae (Phlebotomus sp e Lutzomyia $s p$ ), podendo apresentar diferentes formas clínicas, dependendo da espécie envolvida e da relação do parasita com seu hospedeiro (GONTIJO, 2004; NEVES, 2004).

São protozoários tripanossomatídeos, parasitas intracelulares obrigatórios sob a forma aflagelada ou amastigota presentes no Sistema Fagocítico Mononuclear (SMF) do hospedeiro vertebrado que, uma vez estando no tubo digestivo do vetor se diferenciam na forma promastigota após a picada da fêmea do L. Longipalpis no hospedeiro vertebrado infectado, retirando com sangue ou com a linfa intesticial as leishmanias, que passarão a evoluir e seguindo-se desta maneira o ciclo evolutivo da doença (BRASIL, 2000; 2012).

No novo Mundo, o agente causador é a Leishmania chagasi (L. Chagasi), que é considerado idêntico a Leishmania infantum (L. infantum) do velho mundo (PIMENTEL, 2014).
No hospedeiro vertebrado as formas amastigota de L. Chagasi, são encontradas principalmente nos macrofágos e orgãos linfóides, como medula óssea, baço, fígado e linfonodos. Raramente podem ser encontrados no interior dos leucócitos, íris, placenta e timo (NEVES, 1998; REY, 1991).

A Organização Mundial de Saúde (OMS) estima que a cada ano 1,3 milhões de novos casos de leishmaniose ocorram, e destes, 20.000 a 30.000 mortes. É altamente endêmica no subcontinente indiano e da África Oriental. Estima-se que a cada ano ocorrem no mundo entre 200.000 e 400.000 novos casos de leishmaniose visceral, mais de 90\% deles em seis países: Bangladesh, Brasil, Etiópia, Índia, Sudão e Sudão do Sul (OMS, 2015).

A leishmaniose Visceral devido a sua incidência torna-se uma das doenças mais importantes da atualidade, atingindo cerca de 65 países, tendo uma ampla distribuição continental: Ásia, Europa, África e nas Américas, onde também é conhecida como Calazar ou Leishmaniose Visceral Americana (LVA) (NEVES, 2004; WERNECK, 2010).

Nas Américas, 12 países já reportaram casos autóctones de Leishmaniose Visceral, e destes, cinco notificaram em 2012 no total de 3.231 casos distribuídos em 781 municípios. Observa-se uma redução de 19,3\% de casos notificados em relação ao total do ano anterior, sendo Brasil o país que concentra 96,5\% (3.118) dos casos, seguido do Paraguai 
2,4\% (76), Argentina 0,7\% (24), Colômbia 0,3\% (9) e México 0,1\% (4) (OPAS, 2015).

No Brasil a Leishmaniose Visceral Humana (LVH) apresenta aspectos socioambientais diferenciados em função de sua ampla distribuição geográfica. Desta maneira, a doença vem sendo relatada em vários municípios brasileiros, distribuída em 19 unidades federadas, sendo possível observar transformações importantes no padrão de transmissão da doença (DANTASTORRES F, BRANDÃO FILHO SP, 2006; MARCONDES, 2013).

Até a década de 90 a Região Nordeste correspondia por $90 \%$ dos casos de LVH do país, a incidência média de casos em outras regiões do Brasil no período de 2000-2013, foi caracterizada pelos seguintes coeficientes: Norte $(3,9)$, Sudeste $(0,7)$ e Centro Oeste $(2)$, representando um problema de saúde pública (PIMENTEL, 2014; BRASIL, 2000)

Os dados epidemiológicos dos últimos quinze anos revelam a periurbanização e a urbanização da LVH, destacando-se os surtos ocorridos no estado do Rio de Janeiro, Minas Gerais, São Paulo, Pará, Piauí, Rio Grande do Norte, Bahia, Tocantins e no Mato Grosso do Sul. A doença que era eminentemente rural atualmente passa por um processo de urbanização (NEVES, 2004; OLIVEIRA, 2014; RATH, 2003).

Desta maneira, as doenças negligênciadas se enquadram em um perfil caracterizado pela migração, ocupação urbana precária e irregular, saneamento básico insuficiente e carências nutricionais, alguns dos muitos determinantes de sua ocorrência (WERNECK, 2010).

No Estado de Pernambuco, a situação não é diferente. Logo, seja no ambiente rural, como no urbano, a LV amplia suas áreas de ocorrência, ultrapassando antigos limites geográficos delimitados e tornando-se presente em praticamente toda extensão territorial do estado de Pernambuco (DANTAS-TORRES F, BRANDÃO FILHO SP, 2006).

Dentre o número de casos no País, Pernambuco concentra uma grande casuística da doença, sendo registrados 1.465 casos no período de 2000 a 2010 somente no Estado de Pernambuco e segundo o Ministério da Saúde, um número de óbitos de 122 casos no período de 2000 a 2013 (BRASIL, 2012; 2015).

Ao avaliar as ações desempenhadas para o controle desta endemia é possível observar que ela está essencialmente ligada a três partes relevantes do programa de controle da Leishmaniose Visceral (PCLV): vetor, reservatório e o homem. As ações de controle estão dirigidas à população humana a fim de evitar os riscos de transmissão, ao vetor (levantamento entomológico) e ao reservatório canino, através do inquérito sorológico com a eutanásia dos cães sororreagentes (BRASIL, 2008; NEVES, 2004). 
É preciso levar em conta que são múltiplos os fatores que influenciam a dinâmica das doenças transmitidas por vetores. Além dos fatores ambientais, destacam-se os sócio-demográficos, os biológicos, os médico-sociais, a efetividade dos sistemas locais de saúde, os programas específicos de controle e a história da doença (BARCELLOS, 2009).

De acordo com a Portaria $\mathrm{n}^{\circ} 1.271 \mathrm{DE}$ 06 de Junho de 2014, do Ministério da Saúde, todos os casos de Leishmaniose Visceral Humana são de notificação obrigatória, devendo a sua investigação ser realizada em até 48 horas, e o caso encerrado em até 60 dias, no Sistema de Informações de Agravos de Notificação Compulsória (SINAN-NET). O objetivo é reduzir os índices da doença, realizar o diagnóstico precoce dos casos e desencadear medidas de prevenção e controle em momento oportuno (BRASIL, 2014).

Um dos fatores que contribuem para a manutenção da LVH nos níveis atuais é o desconhecimento da população sobre a doença, os sinais e os sintomas, que se desenvolvem principalmente em crianças desnutridas e pessoas não tratadas. Diante disso, o objetivo deste trabalho foi realizar um levantamento epidemiológico da Leishmaniose humana no Estado de Pernambuco, em uma série histórica de dez anos (2004-2013), demonstrando os Coeficientes Incidências (CI) dos casos humanos e sua distribuídos nas 12 Regiões de Saúde do Estado de Pernambuco.

\section{METODOLOGIA}

O Estado de Pernambuco possui uma área aproximadamente de $98.146,315 \mathrm{Km}^{2}$, com uma população estimada em 9.277.527 habitantes segundo o Instituto Brasileiro de Geografia e Estatística (IBGE), distribuída em 185 municípios localizados em 12 Regiões de Saúde, dos quais 125 são endêmicos para Leishmaniose Visceral Humana (LVH). O presente estudo, de caráter descritivo, foi desenvolvido, buscando conhecer o coeficiente incidência dos casos humanos notificados e confirmados para LVH. Os dados foram obtidos do Sistema de Informação de Agravos de Notificação Compulsória (SINAN -NET) no período de 2004 a 2013, notificados e confirmados pela rede de saúde dos municípios que compõem as regiões de saúde de Pernambuco. Após a obtenção dos dados do SINAN-NET através do aplicativo TABWIN (DATASUS), foram construídos gráficos, sendo calculado os coeficientes de incidência para Leishmaniose Visceral Humana (LVH), que demonstraram a situação epidemiológica deste agravo nas Regiões de Saúde em estudo. O Coeficiente de Incidência foi calculado tendo como base o número de casos novos avaliados em determinada área e período, multiplicado por 100.000, dividido pela população residente na área e período considerado. 


\section{RESULTADOS}

De acordo com informações constantes no banco de dados do Sistema de Informação de Agravos de Notificação Compulsória (SINAN-NET), disponibilizado pela Secretaria Estadual de Saúde de Pernambuco, foram notificados $2.255 \mathrm{e}$ confirmados 863 casos de Leishmaniose Visceral Humana (LHV) no período de 2004 a 2013. Nos dois primeiros anos do estudo (2004-2005) o estado de Pernambuco apresentou uma incidência de 2,23 casos/100.000 habitantes. No final da série histórica (2012 e 2013), o coeficiente declinou para 1,49 casos/100.000 habitantes. $\mathrm{O}$ coeficiente de incidência para LVH apresentou o menor índice no ano de 2013 (0,72 casos/100.000 habitantes), sendo o maior registro em 2004 (1,16 casos/100.000 habitantes), com 108 casos novos confirmados (figura 1).

Comparando-se os casos de LVH em estudo, observaram-se os maiores coeficientes de incidência na IV, VII, VIII e IX Regiões de Saúde, nos anos de 2004, 2006, 2010, 2012 e $2013 \quad(2,94, \quad 8,27, \quad 6,89, \quad 5,04 \quad 5,79$, casos/100.000 habitantes respectivamente), sendo o menor índice registrado na I Região de Saúde (0,07/100.000 habitantes, no ano de 2008. Com exceção da II, III, VII, IX, X e a XI Regiões de Saúde, as demais apresentaram um declínio de casos notificados no último ano do estudo. Na I e II Região de Saúde apresentou oscilações no aumento e no declínio nos coeficientes em todo período estudado. No que diz respeito a III Região de Saúde, destaca-se o ano de 2004, onde não houve registro de casos confirmados, como também as V e a XII no ano de 2013. A IV Região de Saúde registrou 178 casos nos anos em estudo, com maiores incidências em 2004, 2005 e 2008 (2,94; 1,51; 2,26 casos/100.000 habitantes, respectivamente). Com exceção do número elevado de casos confirmados na IV Região de Saúde, houve diminuições nas incidências, em detrimento a V e VI Regiões de Saúde nos anos de 2004 (0,93; 0,48 casos/100.000 habitantes), 2007 (0,18;0,97 casos/100.000 habitantes e $2008(0,37 ; 0,73$ casos/ 100.000 habitantes respectivamente). As incidências identificadas na VII e XI Região de Saúde apresentaram variações nos anos avaliados, tendo picos registrados em 2006 e 2010. A VIII Região de Saúde registrou sua menor incidência no ano de 2009 (3,36 caso/100.000 habitantes), sendo que nos anos de 2012 a 2013 apresentou um coeficiente de incidência acima de 4,83/100.000 habitantes. A XI Região de Saúde apresentou sua maior incidência em 2004 e $2010 \quad(2,98 / 100.000$ habitantes respectivamente) e tal coeficiente supera qualquer um dos observados na $\mathrm{X}$ Região de Saúde. Salienta-se que nos anos de 2005, 2008 e 2009 não há registro de casos na X Região de Saúde. 
FIGURA 1: Coeficiente de Incidência (CI) dos casos de Leishmaniose Visceral no estado de Pernambuco, no período de 2004 a 2013.

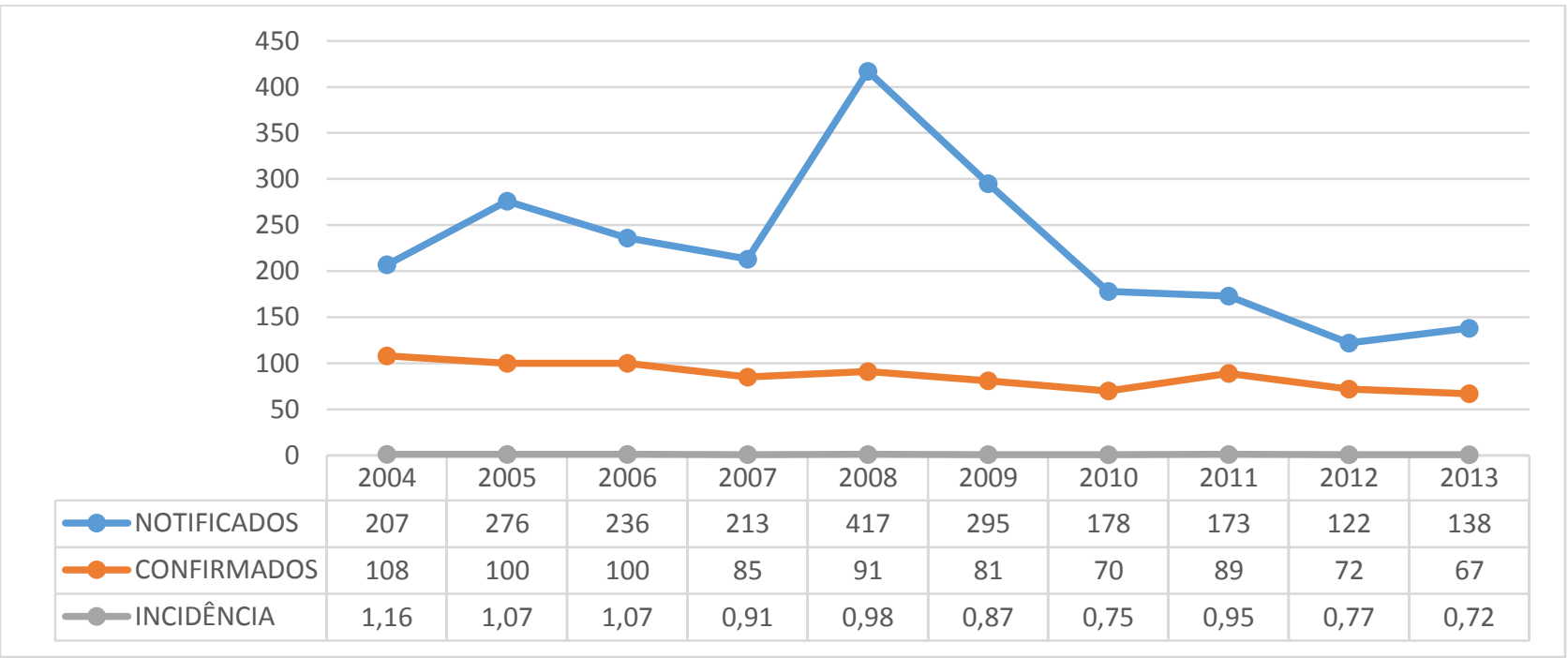

FIGURA 2: Coeficiente de Incidência (CI) dos casos de Leishmaniose Visceral da I GERES, no período de 2004 a 2013.

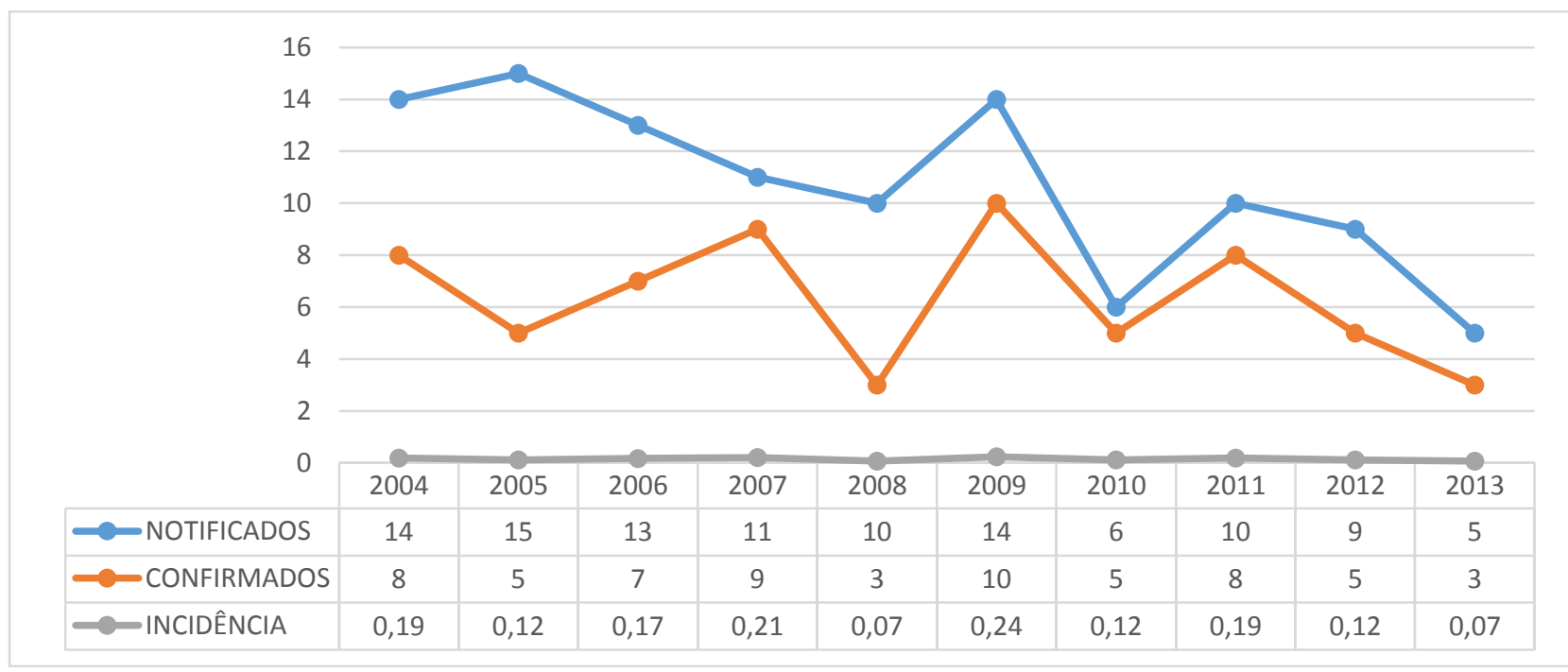

FIGURA 3: Coeficiente de Incidência (CI) dos casos de Leishmaniose Visceral da II GERES, no período de 2004 a 2013.

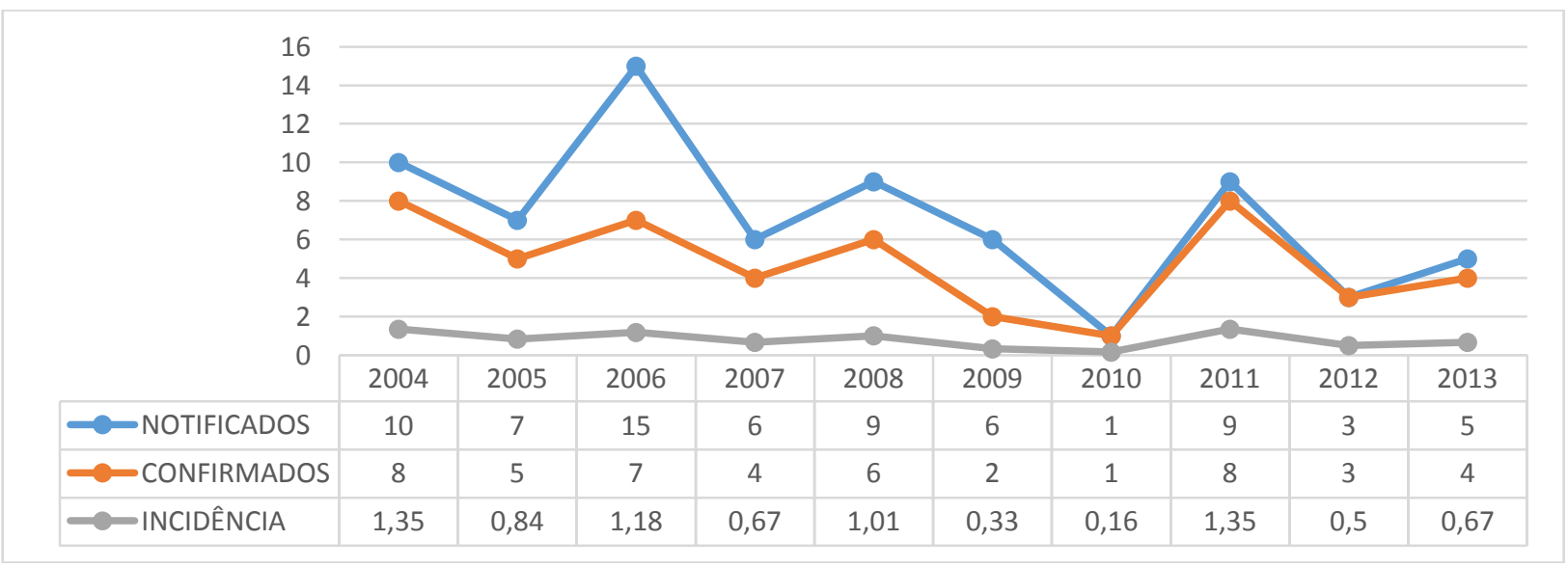


FIGURA 4: Coeficiente de Incidência (CI) dos casos de Leishmaniose Visceral da III GERES, no período de 2004 a 2013.

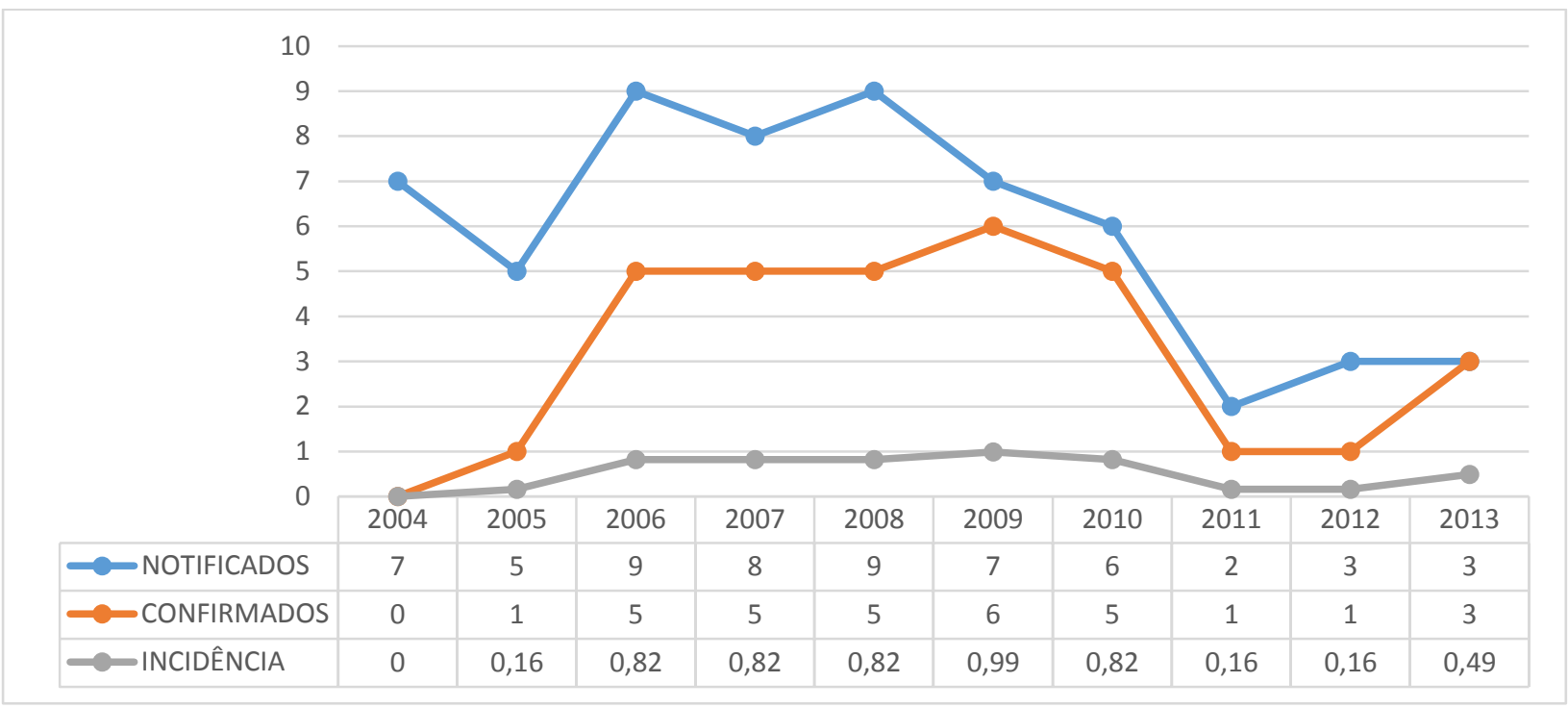

FIGURA 5: Coeficiente de Incidência (CI) dos casos de Leishmaniose Visceral da IV GERES, no período de 2004 a 2013.

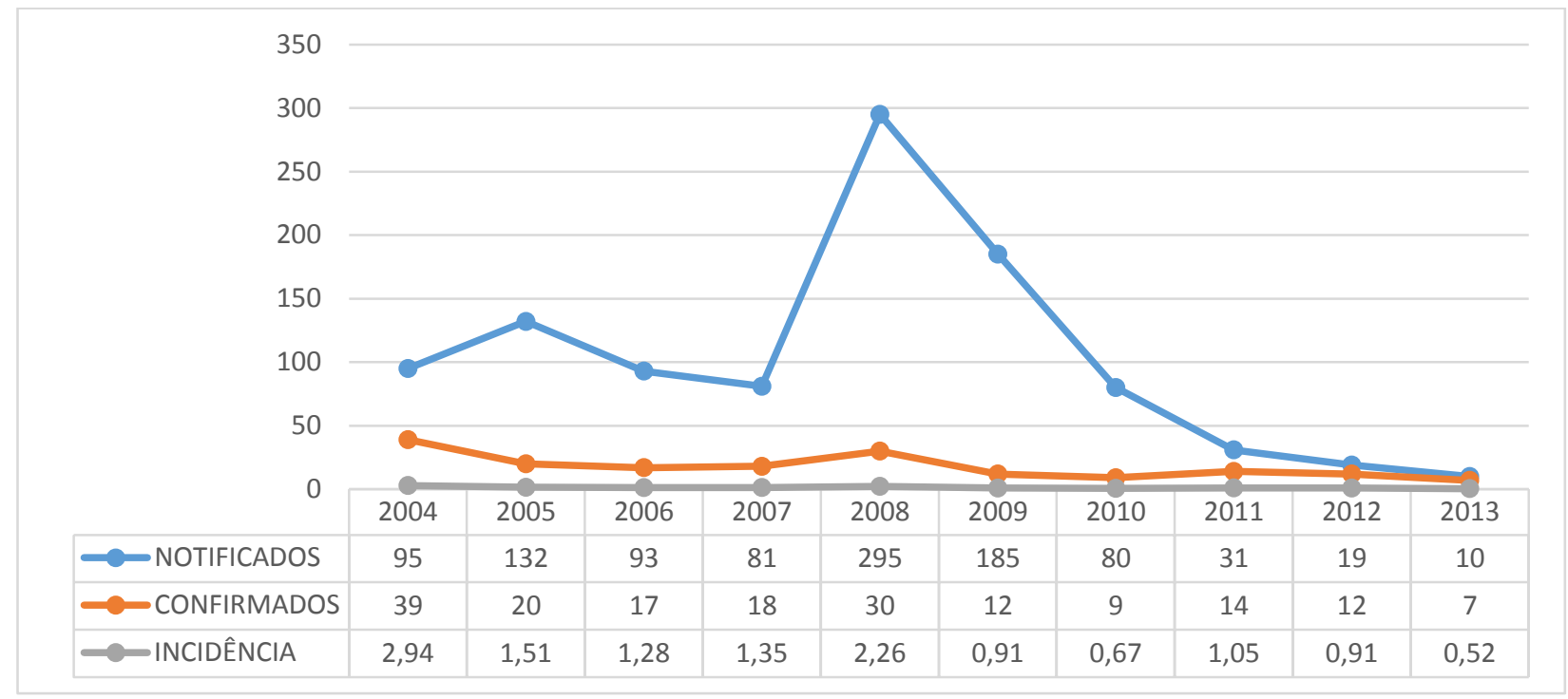

FIGURA 6: Coeficiente de Incidência (CI) dos casos de Leishmaniose Visceral da V GERES, no período de 2004 a 2013.

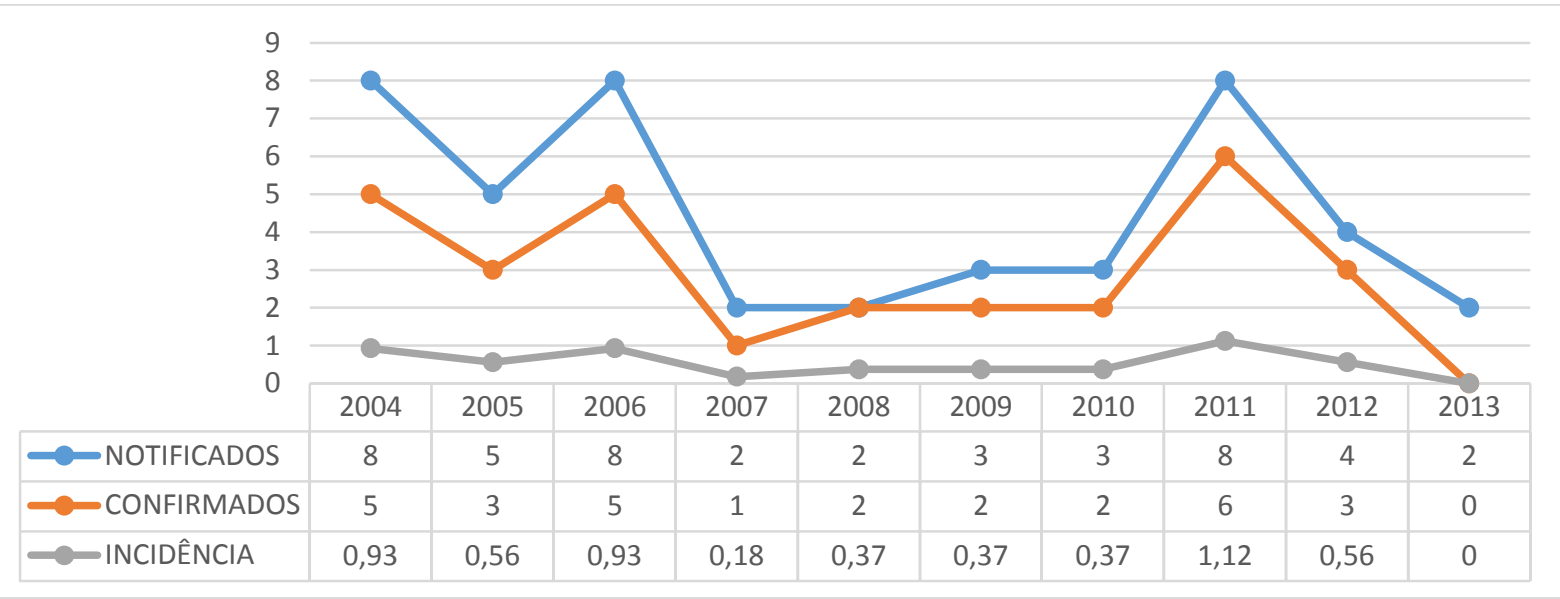


FIGURA 7: Coeficiente de Incidência (CI) dos casos de Leishmaniose Visceral da VI GERES, no período de 2004 a 2013.

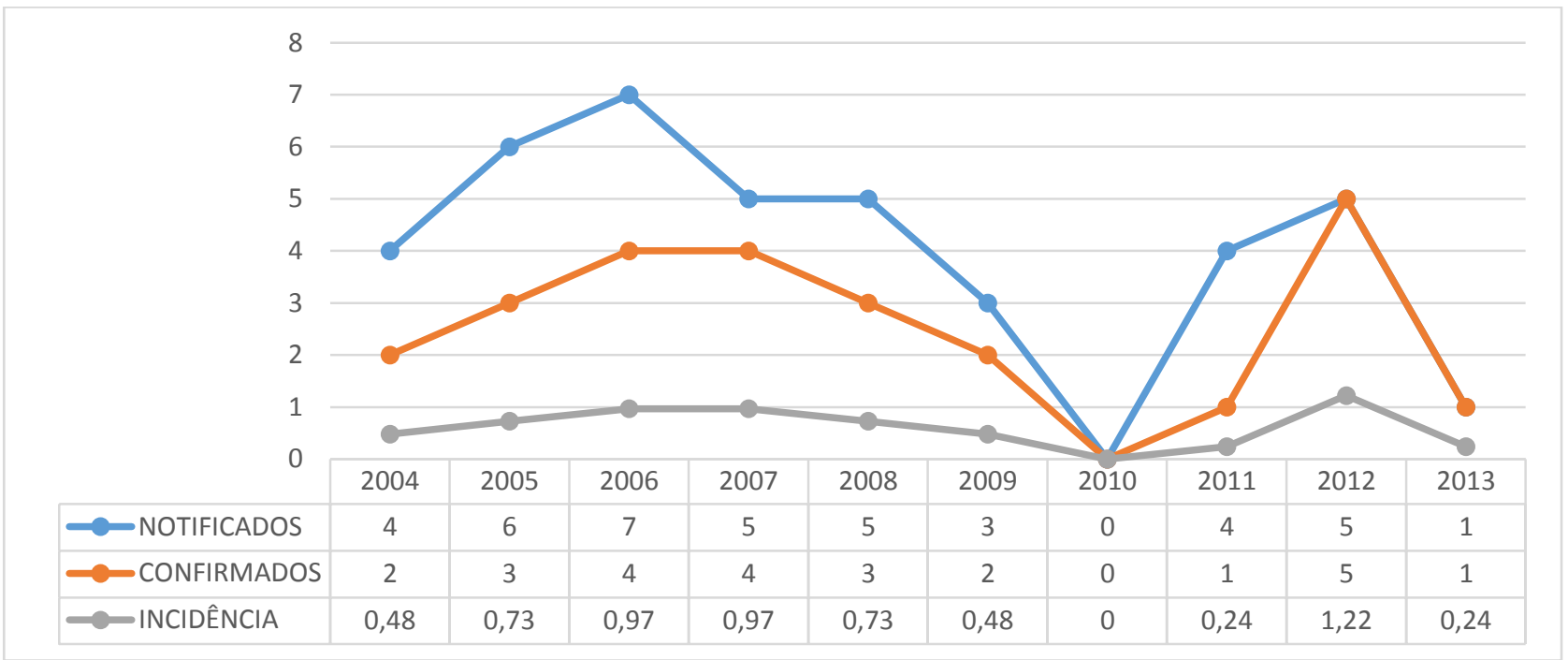

FIGURA 8: Coeficiente de Incidência (CI) dos casos de Leishmaniose Visceral da VII GERES, no período de 2004 a 2013.

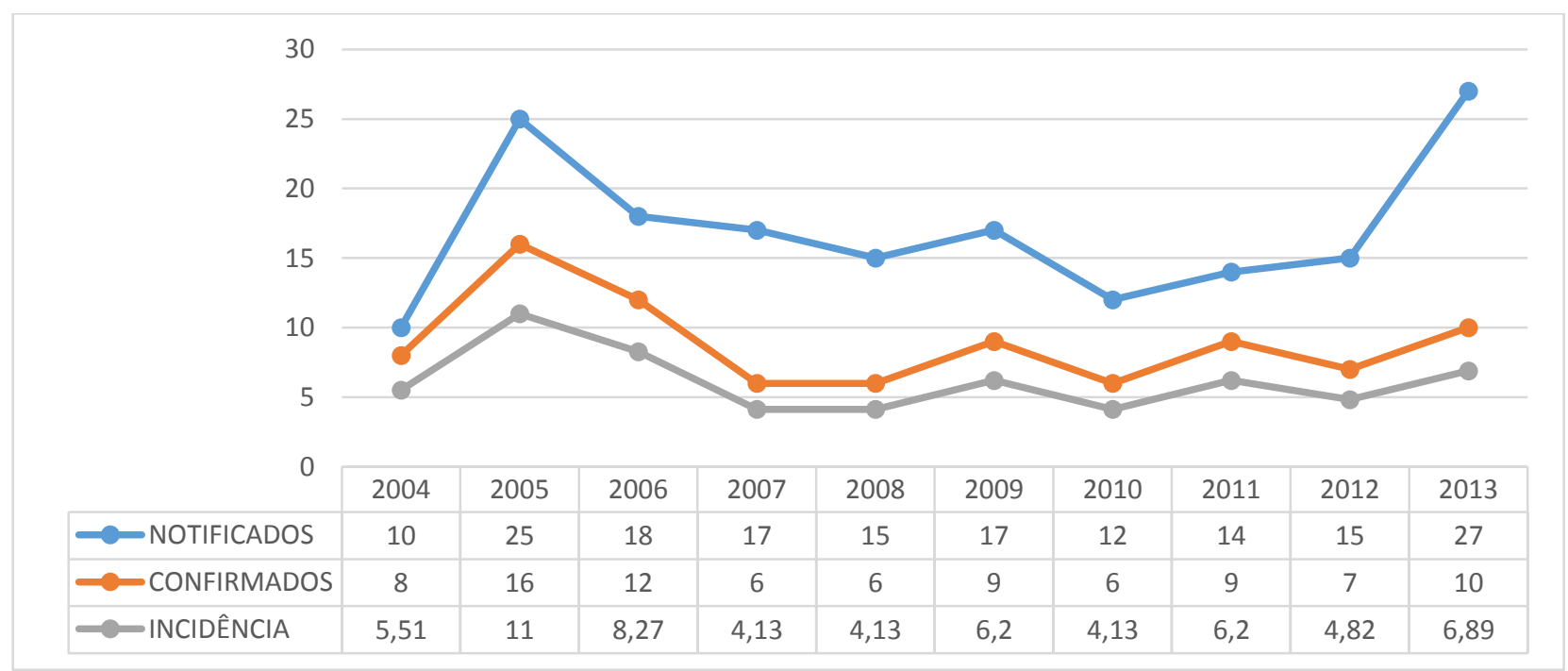

FIGURA 9: Coeficiente de Incidência (CI) dos casos de Leishmaniose Visceral da VIII GERES, no período de 2001 a 2013.

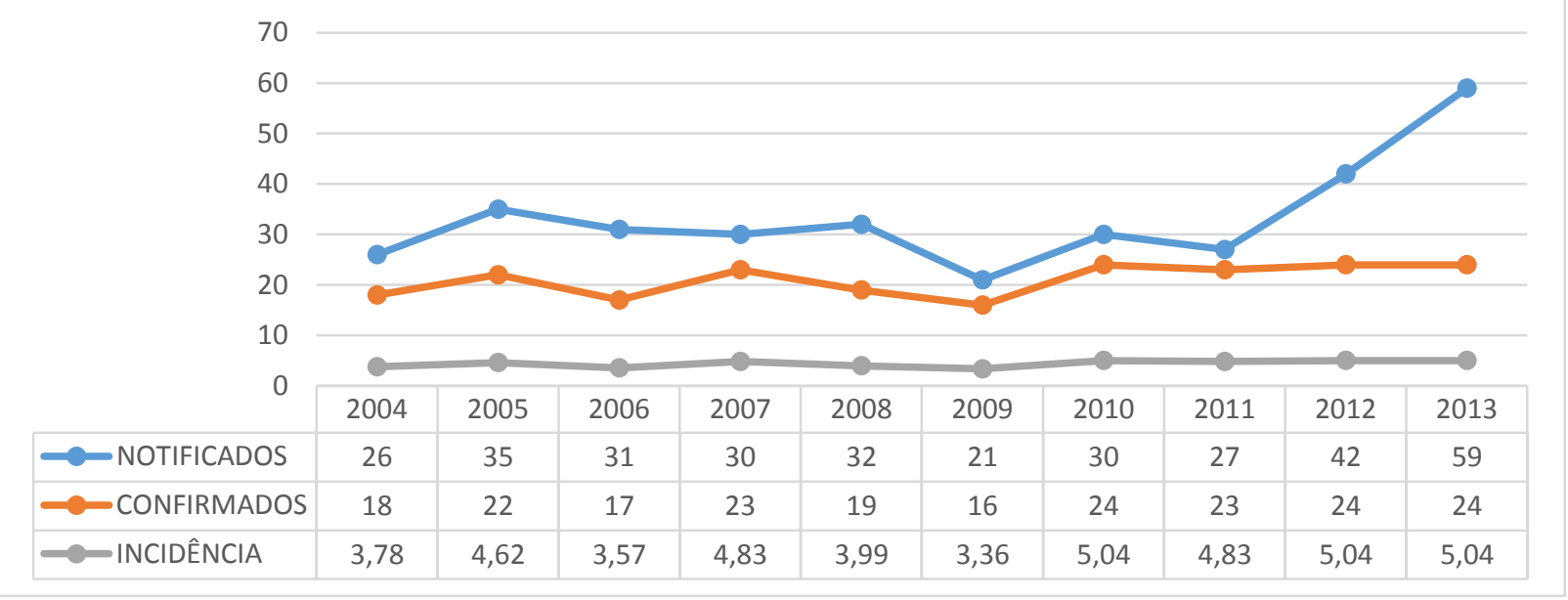


FIGURA 10: Coeficiente de Incidência (CI) dos casos de Leishmaniose Visceral da IX GERES, no período de 2001 a 2013.

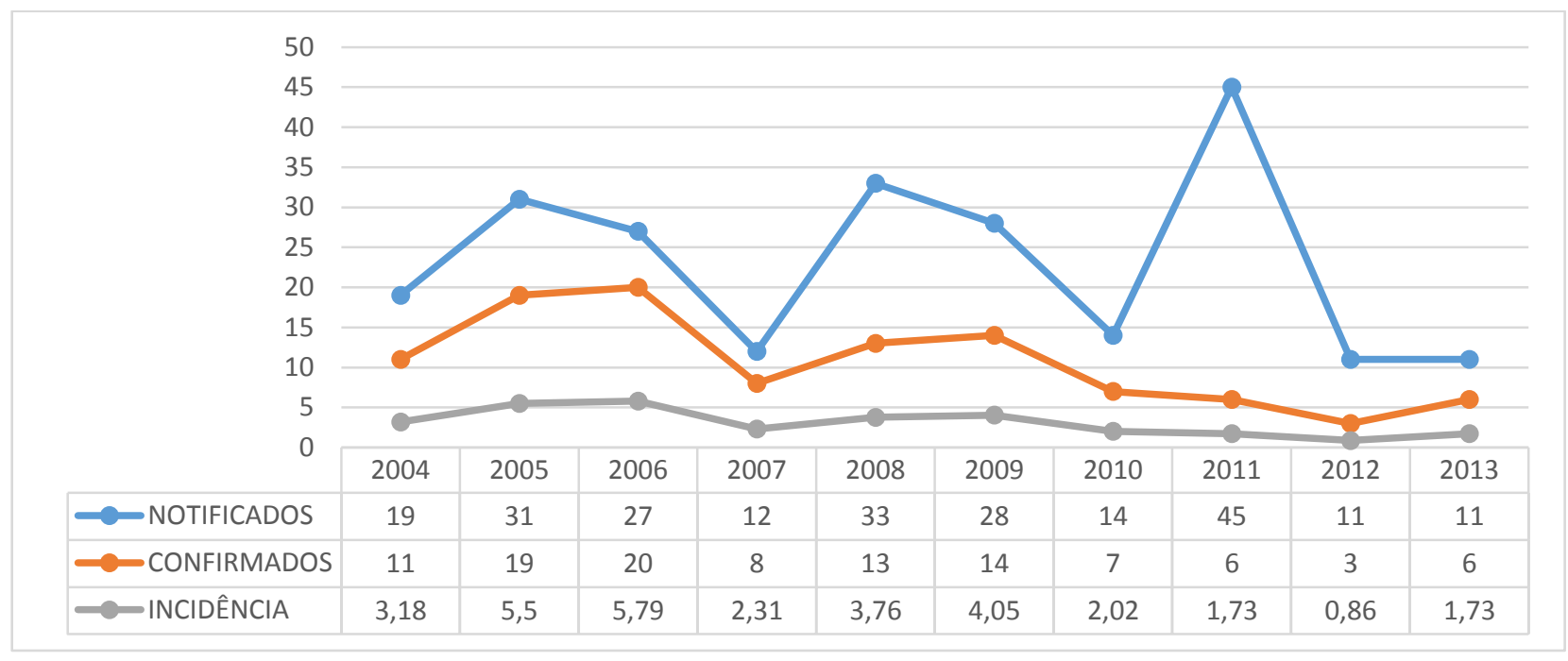

FIGURA 11: Coeficiente de Incidência (CI) dos casos de Leishmaniose Visceral da X GERES, no período de 2001 a 2013.

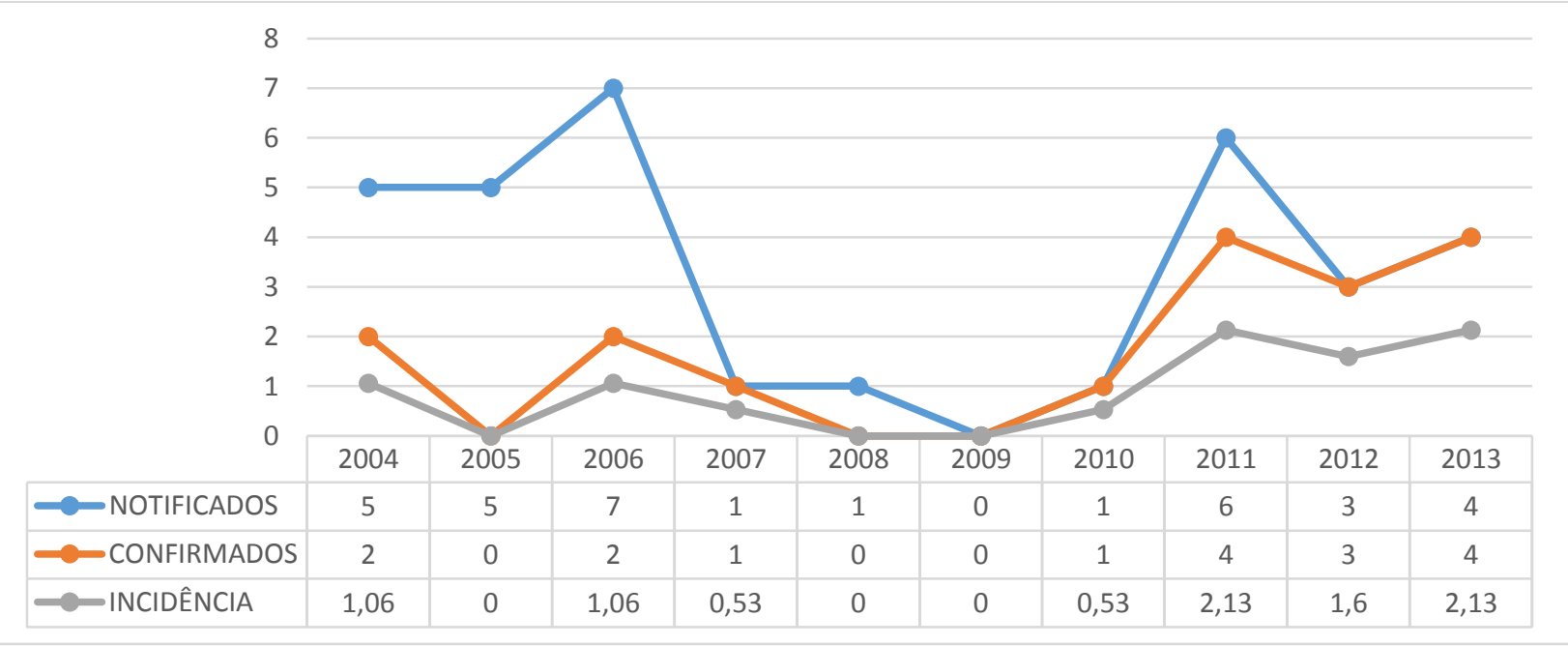

FIGURA 12: Coeficiente de Incidência (CI) dos casos de Leishmaniose Visceral da XI GERES, no período de 2001 a 2013.

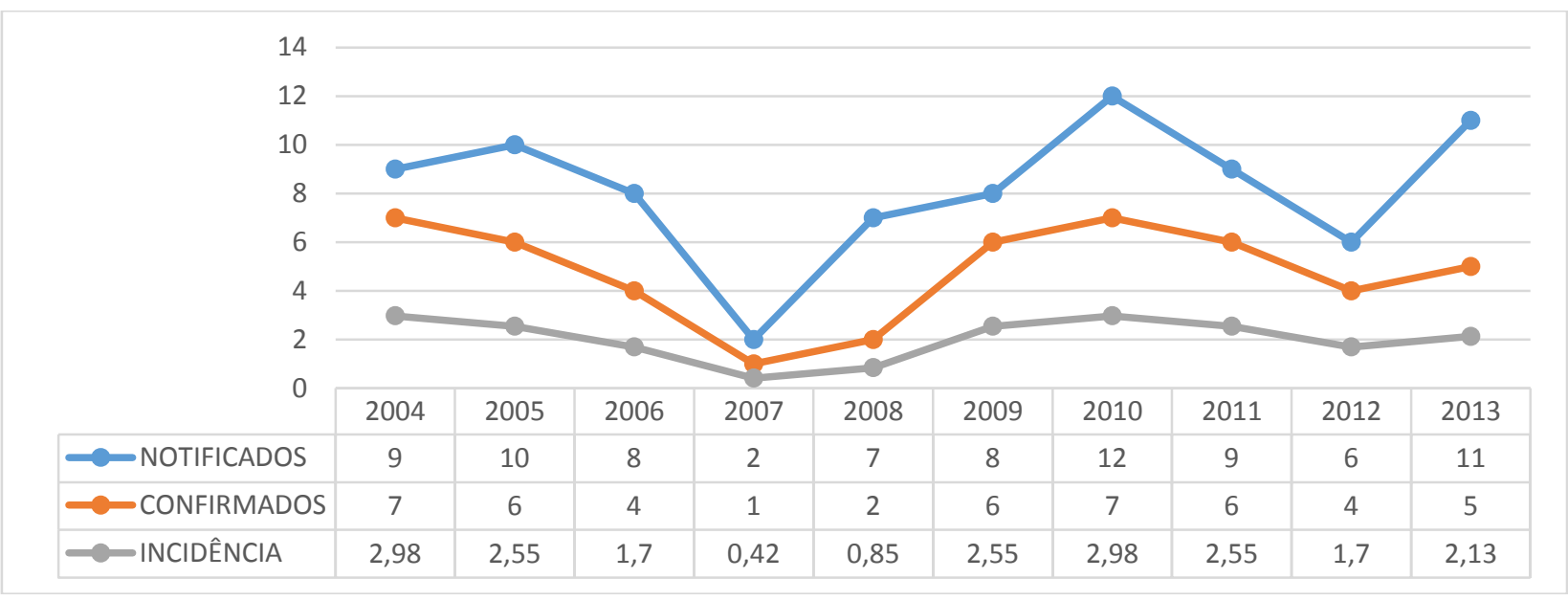


FIGURA 13: Coeficiente de Incidência (CI) dos casos de Leishmaniose Visceral da XII GERES, no período de 2001 a 2013.

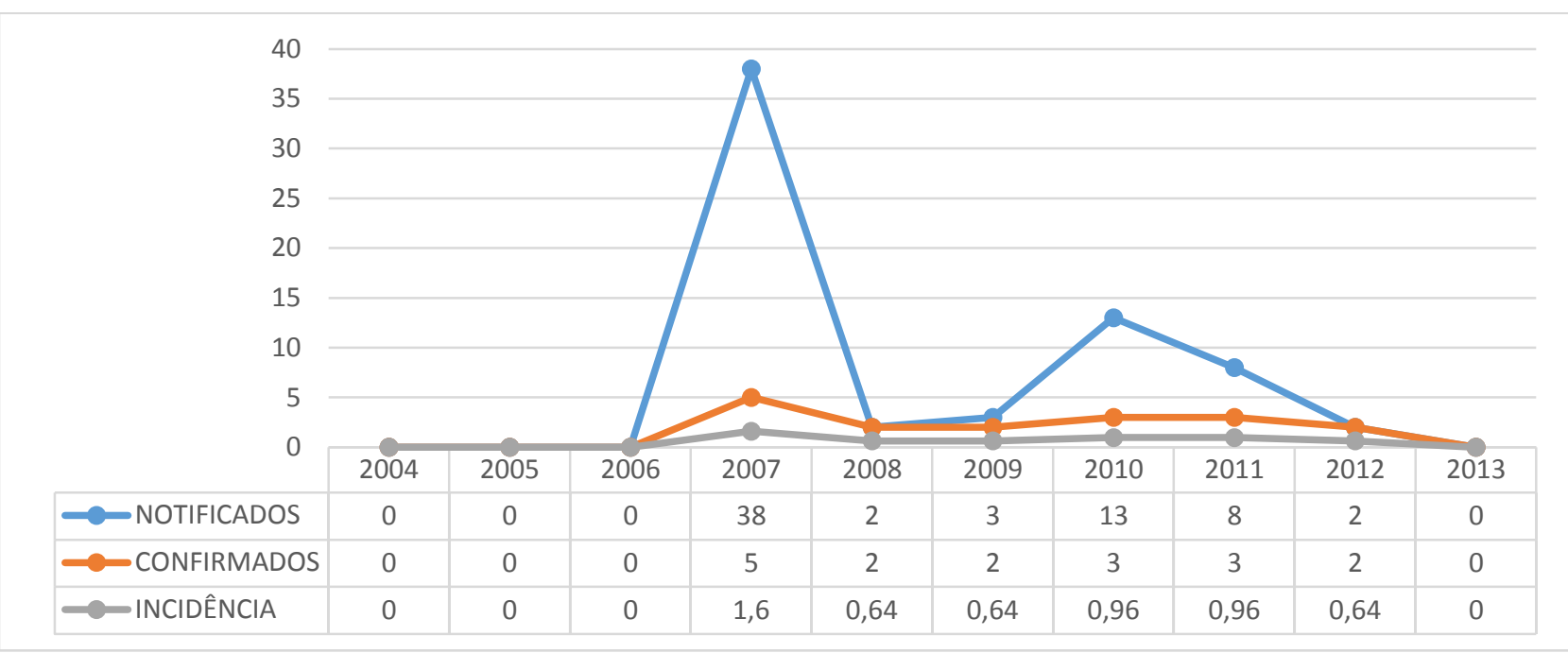

\section{DISCUSSÃO}

Os estudos epidemiológicos de campo para $\mathrm{LVH}$, expressam a melhor maneira de avaliar a epidemiologia deste agravo, porém o alto custo e o tempo de coleta das informações juntamente com o emprego de sua aplicação, se tornam impraticável. Dessa maneira os dados provenientes dos sistemas de informações oficiais do Ministério da Saúde, apesar de certas limitações tem sido uma ferramenta amplamente utilizada, gerando informações importantes para compreensão da LVH ( SILVA NUNES, 2008). O ciclo de transmissão desta endemia presente anteriormente quase que exclusivamente em ambientes rurais e silvestres, passou a existir com grande frequência nos centros urbanos (RATH, 2003).

A LVH é uma doença negligenciada e que nos últimos anos é possível perceber mudanças significativas na organização rural no país, tendo como consequência a migração da população para os grandes centros, resultando em condições precárias de vida (pobreza, desnutrição, saneamento básico insuficiente), caracterizando-se como fatores decisivos que favorecem a expansão da doença (SCANDAR, 2011; BEVILACQUA PD, 2001; TORRES, F, 2006).

$\mathrm{O}$ contato ente o homem e o reservatório canino, bem como, o crescimento da população vetorial, estão diretamente ligados ao ciclo da doença e são determinantes na disseminação desta endemia. Nesse contexto, o cão (canis familiares) constitui um papel importante na transmissão, pois participa do cotidiano das cidades e do campo, sendo responsável pela manutenção do foco. Com a migração da população do campo para a cidade ou a migração de habitantes de cidades endêmicas para áreas não endêmicas, parece constituir 
um risco para propagação da doença. Devendo ser alvo do controle rigoroso da vigilância em saúde, visto que, não há vacinas para população canina (MELO, 2004; TORRES, F. 2006).

Segundo o Ministério da Saúde o tratamento não é recomendado para os casos caninos, pois não diminui a sua importância como reservatório do parasita e não existem evidências adequadas que mostrem a eficácia da vacina na prevenção da infecção na população canina, bloqueando a transmissão através do vetor em cães previamente vacinados (WERNECK, 2008).

Para a LVH, é necessário que o Estado e os municípios, estejam com suas equipes de saúde médicos, enfermeiros, agentes de endemias, agente comunitários, bem como, os equipamentos de saúde (hospitais e laboratórios) que garantam o diagnóstico precoce dos casos, a realização dos diagnósticos preconizados pelo Ministério da Saúde, visando a confirmação dos casos, o tratamento em tempo oportuno evitando o óbito.

No que tange ao controle do vetor, a instância estadual deve considerar experiências desenvolvidas, como a estratificação das áreas através do levantamento entomológico, classificando-as em vulnerável não receptiva e vulnerável receptiva e o controle químico por meio da utilização de inseticidas de ação residual, medida essa, recomendada no âmbito da proteção coletiva. Esta ação é realizada para o inseto em sua fase adulta, com o objetivo de evitar ou eliminar o contato entre o vetor e a população humana (GONTIJO, 2004).

Com o presente estudo foi possível observar que a Leishmaniose Visceral Humana está presente em todo território pernambucano, da I a XII Região de Saúde, estabelecida em áreas rurais e urbanas, tendo os seus maiores coeficientes de incidência no Agreste e no sertão pernambucano, representando um problema de saúde pública.

O Programa de Controle da Leishmaniose Visceral (PCLV) precisa de investimentos através de políticas públicas que fortaleçam os seus componentes que são caracterizados pelo diagnóstico clínico laboratorial, tratamento, vigilância epidemiológica, prevenção e medidas de controle, visando também a redução das desigualdades às quais as populações das regiões de saúde vêm sendo submetidas ao longo dos anos. Se não houver uma intervenção no controle a esta endemia, a LVH poderá migrar para as regiões com maior densidade populacional, mudando, inclusive, seu padrão hoje basicamente restrito às populações de baixo poder aquisitivo.

Considerando os resultados encontrados, faz-se necessário o desenvolvimento de um plano de intervenção, que envolva a rede de saúde em todos os níveis de complexidade para o diagnóstico, 
acompanhamento, o tratamento dos casos, a notificação e o encaminhamento, quando necessário, através do estabelecimento de um protocolo de referência e contra referência municipal, estadual, interestadual e federal para os casos mais complexos de LVH.

Outras estratégias passam pelo envolvimento da população, que deve ser empoderada para agir como instrumento de controle dessa endemia e para tal precisa conhecê-la (risco de contrair a Leishmaniose, ciclo biológico, as consequências, gravidade e tratamento) e pelo desenvolvimento de atividades de educação em saúde, através do envolvimento efetivo das equipes multiprofissionais das secretarias de saúde, bem como, interinstitucionais (Secretaria de obra, Meio Ambiente, Centros de Controle de Zoonoses) com vistas no trabalho articulado entre as diferentes instância do poder público.

\section{REFERÊNCIAS}

BARCELLOS, C.; MONTEIRO, A.M.V.; CORVALÁN, C.; GURGEL, H.C.; CARVALHO, M.S.; ARTAXO, P.; HACON, S.; RAGONI, V. Mudanças climáticas e ambientais e as doenças infecciosas: cenários e incertezas para o Brasil. Epidemiologia e Serviços de Saúde, Brasília, v.18, n.3, p.285304, 2009

BRASIL. Ministério da Saúde. Extraído de [http://portalsaude.saude.gov.br/images/pdf/2 014/setembro/09/LV-Coef-Incid--ncia.pdf], acesso em [03 de agosto de 2015].

BRASIL. Ministério da Saúde, Secretaria de Vigilância em Saúde. Casos confirmados de Leishmaniose Visceral: Brasil, grandes regiões e unidades federadas (1990 a 2010). Disponível em:

http://portal.saude.gov.br/portal/arquivos/pdf/l v_casos_05_09_11.pdf. Acesso em: 8 de novembro de 2012.

BRASIL. Ministério da Saúde. Secretaria de Vigilância em Saúde. Departamento de Vigilância Epidemiológica. Doenças infecciosas e parasitárias: guia de bolso. $7^{\text {a }}$ ed. Brasília; 2008.

BRASIL. Ministério da Saúde. Mudanças climáticas e ambientais e seus efeitos na saúde: cenários e incertezas para o Brasil. Organização Pan Americana da Saúde, 2008.

BRASIL. Ministério da Saúde. Portaria 1.271 de 06 de junho de 2014. Diário Oficial da União, Brasília, 2014.

BEVILACQUA PD, Paixão HH, Modena CM, Castro MCPS. Urbanização da leishmaniose visceral em Belo Horizonte. Arq Bras Med Vet Zootec. 2001;53(1):1-8

\section{DANTAS-TORRES F, BRANDÃO FILHO}

SP. Expansão geográfica da leishmaniose visceral no estado de Pernambuco. Revista da Sociedade Brasileira de Medicina Tropical 39:353-35, 2006.

GONTIJO, C.M.F.; Melo, M.N.

Leishmaniose Visceral no Brasil: quadro atual, desafios e perspectivas. Revista Brasileira de Epidemiologia 2004; 7(3):338349.

MARCONDES, M. \& Rossi, C. N. (2014) Visceral leishmaniasis in Brazil. Brazilian Journal of Veterinary Research and Animal Sciencen 2013; 50(5): 341-352.

NEVES, V.; Silva, L. As Leishmanioses, Uma Visão Para o Clínico. Revista Prática Hospitalar 2004; 4(36): 45-49.

MELO, M. N. Leishmaniose visceral no Brasil: desafios e perspectivas. Revista Brasileira de Parasitologia Veterinária, v. 23, sup. 1, p. 41-45, 2004 
NEVES, D.P. Parasitologia humana. $9^{\mathrm{a}}$ ed. São Paulo: Atheneu; 1998.

OLIVEIRA, I. B. \& Bucar et al.

Epidemiological and environmental aspects of visceral leishmaniasis in children under 15 years of age between 2007 and 2012 in the City of Araguaína, State of Tocantins, Brazil. Rev. Soc. Bras. Med. Trop., Uberaba, v. 47, n. 4, p. 476-482, ago. 2014

OMS, Organización Mundial de la Salud. Nota descriptiva N³75 Febrero de 2015. Disponível em: http://www.who.int/mediacentre/factsheets/fs 375/es/ Acesso em: 03 agosto. 2015.

OPAS, Organización Panamericana de la Salud. Leishmaniosis- Informe Epidemiológico de las Américas. Dispónivel em:

[ http://www.paho.org/hq/index.php?option=c om topics\&view=article\&id=29\&Itemid=407 54] Acesso em: 03 agosto. 2015.

PIMENTEL, M. High visceral leishmaniasis mortality rate in Barra Mansa, a new area of visceral leishmaniasis transmission in the State of Rio de Janeiro, Brazil. Rev. Soc. Bras. Med. Trop., Uberaba, v. 47, n. 4, p. 521523, ago. 2014.

RATH, S., TRIVELIN, L.A., IMBRUNITO, T.R., TOMAZELA, D.M., JESUS, M.N., MARZAL, P.C. et al. Antimoniais empregados no tratamento da leishmaniose: estado da arte. Quim Nova 2003; 26:550-553

REY, L. Parasitologia: parasitos e doenças parasitárias do homem nas Américas e na áfrica. $2^{\mathrm{a}}$ ed. Rio de Janeiro: Guanabara Koogan; 1991.

SCANDAR SAS, Silva RA, Cardoso Jr RP, Oliveira FH. Ocorrência de leishmaniose visceral americana na região de São José do Rio Preto, estado de São Paulo, Brasil. Bol Epidemiol Paulista. 2011;8(88):13-22.
Tegumentar e descrição das populações de flebotomíneos no município de Acrelândia, Acre, Brasil. Rev Bras Epidemiol. 2008;11(2):241-51.

TORRES, F.F.S. Expansão geográfica da leishmaniose visceral no Estado de Pernambuco. Revista da Sociedade Brasileira de Medicina Tropical 2006; 39(4):352-356

WERNECK, G. L. Expansão geográfica da leishmaniose visceral no Brasil.Cad. Saúde Pública, Rio de Janeiro, v. 26, n. 4, p. 644645, Apr. 2010. WERNECK, G.L., PEREIRA, T.J.C.F., FARIAS, G.C., SILVA, F.O., CHAVES, F.C., GOUVÊA, M.V. et al. Avaliação das estratégias de controle da leishmaniose visceral na cidade de Teresina, Estado do Piauí, Brasil: resultados do inquérito inicial 2004. Epidemiol Serv Saúde. 2008; 17(2):8796.

SILVA-NUNES M, Cavasini CE, Silva NS, Galati EAB. Epidemiologia da Leishmaniose 